Research Article

\title{
Some Bohr-Type Inequalities for Bounded Analytic Functions
}

\author{
Hong Wang (iD \\ School of Big Data and Artificial Intelligence, Chizhou University, Chizhou 247000, China \\ Correspondence should be addressed to Hong Wang; hongw@czu.edu.cn
}

Received 8 September 2021; Accepted 20 October 2021; Published 23 November 2021

Academic Editor: Ming-Sheng Liu

Copyright (C) 2021 Hong Wang. This is an open access article distributed under the Creative Commons Attribution License, which permits unrestricted use, distribution, and reproduction in any medium, provided the original work is properly cited.

In this paper, some new versions of Bohr-type inequalities with one parameter or involving convex combination for bounded analytic functions of Schwarz function are established. Some previous inequalities are generalized. All the results are sharp.

\section{Introduction and Preliminaries}

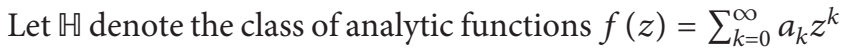
defined in the unit disk $\mathbb{D}:=\{z \in \mathbb{C}:|z|<1\}$ such that $|f(z)|<1$ in $\mathbb{D}$. The Bohr's theorem states that if $f(z)=$ $\sum_{k=0}^{\infty} a_{k} z^{k} \in \mathbb{H}$, then

$$
\sum_{k=0}^{\infty}\left|a_{k} \| z\right|^{k} \leq 1, \quad \text { for }|z| \leq \frac{1}{3}
$$

the value $1 / 3$ is the best possible, and the inequality (1) is called classical Bohr inequality. In 1914, Bohr originally established the inequality (1) for $|z|<1 / 6$ [1]. But subsequently later, Wiener, Riesz, and Schur independently proved the inequality (1) for $|z|<1 / 3$, and the value is proved to be sharp [2-4].

Bohr phenomenon and Bohr radius problem are very interesting. Many generalized forms are studied and a lot of Bohr-type inequalities are obtained. Kayumov considered the Bohr-type inequalities for odd analytic functions and some other classes of analytic function in [5]. Bhowmik and Das [6] studied Bohr's phenomenon for subordinating families of certain univalent functions. Huang et al. [7] refined the Bohr inequality by allowing Schwarz function in place of the initial variable of the functions. Hu et al. [8, 9] established some Bohr inequalities with one parameter or involving convex combination. Abu-Muhanna [10] investigated the Bohr phenomenon for the class of analytic functions from the unit disk into the punctured unit disk.
Some authors considered the Bohr phenomenon for functions defined on other domains, such as concave-wedge domain [11, 12], convex domain [13], and the exterior of a compact domain [14].

The Bohr-type inequalities were also considered in other branches of mathematics. The analogous Bohr's radius was also studied for K-quasiconformal harmonic mappings by Liu and Ponnusamy [15]. Djakov and Ramanujan [16], Aizenberg [17], and Bayart et al. [18] generalized the Bohr inequality to the case of higher dimensions. Abu-Muhanna and Gunatillake [19] considered Bohr phenomenon in weighted Hardy-Hilbert spaces. Blasco [20] obtained the Bohr's radius of a Banach space. In [21], Paulsen explored Bohr's radius problem in Banach algebras. For more discussion on the Bohr-type inequalities, we can refer to [22-25].

We recall some results as follows.

Theorem 1 (see [26]). Suppose $f(z)=\sum_{k=0}^{\infty} a_{k} z^{k} \in \mathbb{H}$, $N \in \mathbb{N}=\{1,2,3, \ldots\}$, then

$$
|f(z)|+\sum_{k=N}^{\infty}\left|a_{k} \| z\right|^{k} \leq 1, \quad \text { for }|z| \leq \alpha,
$$

where $\alpha$ is the positive root of the equation

$$
2(1+r) r^{N}-(1-r)^{2}=0 .
$$

The radius $\alpha$ is the best possible. Moreover, 


$$
|f(z)|^{2}+\sum_{k=N}^{\infty}\left|a_{k}\right| r^{k} \leq 1, \quad \text { for }|z| \leq \beta,
$$

where $\beta$ is the positive root of the equation

$$
(1+r) r^{N}-(1-r)^{2}=0 .
$$

The radius $\beta$ is the best possible.

Theorem 2 (see [27]). Suppose $f(z)=\sum_{k=0}^{\infty} a_{k} z^{k} \in \mathbb{H}$, and $s \in \mathbb{N}$, then

$$
|f(z)|+\sum_{k=1}^{\infty}\left|a_{s k} \| z\right|^{s k} \leq 1, \quad \text { for }|z| \leq \gamma,
$$

where $\gamma$ is the positive root of the equation

$$
r^{s+1}+3 r^{s}+r-1
$$

The radius $\gamma$ is the best possible.

Theorem 3 (see [27]). Suppose $f(z)=\sum_{k=0}^{\infty} a_{k} z^{k} \in \mathbb{U}$, then

$$
|f(z)|+\left|f^{\prime}(z)\left\|z\left|+\sum_{k=2}^{\infty}\right| a_{k}\right\| z\right|^{k} \leq 1, \quad \text { for }|z| \leq \frac{\sqrt{17}-3}{4},
$$

where $(\sqrt{17}-3) / 4$ is the best possible.

Theorem 4 (see [28]). Suppose $f(z)=\sum_{k=0}^{\infty} a_{k} z^{k} \in \mathbb{H}$, $a:=\left|a_{0}\right|$, then

$$
\sum_{k=0}^{\infty}\left|a_{k} \| z\right|^{k}+\left(\frac{1}{1+a}+\frac{|z|}{1-|z|}\right) \sum_{k=1}^{\infty}\left|a_{k}\right|^{2}|z|^{2 k} \leq 1, \quad \text { for }|z| \leq \frac{1}{2+a}
$$

and the numbers $1 /(2+a)$ and $1 /(1+a)$ cannot be improved. Moreover,

$$
a^{2}+\sum_{k=1}^{\infty}\left|a_{k} \| z\right|^{k}+\left(\frac{1}{1+a}+\frac{|z|}{1-|z|}\right) \sum_{k=1}^{\infty}\left|a_{k}\right|^{2}|z|^{2 k} \leq 1, \quad \text { for }|z| \leq \frac{1}{2},
$$

and the numbers $1 / 2+a$ and $1 /(1+a)$ cannot be improved.

In order to establish our main results, we need the following lemmas, which will play the key role in proving the main results of this paper.

Lemma 1 (see [27]) (Schwarz-Pick lemma). Suppose $f(z)=\sum_{k=0}^{\infty} a_{k} z^{k} \in \mathbb{H}$, then

$$
\frac{\left|f\left(z_{1}\right)-f\left(z_{2}\right)\right|}{\left|1-\overline{f\left(z_{1}\right)} f\left(z_{2}\right)\right|} \leq \frac{\left|z_{1}-z_{2}\right|}{\left|1-\overline{z_{1}} z_{2}\right|}, \quad \text { for } z_{1}, z_{2} \in \mathbb{D}
$$

and the equality holds for distinct $z_{1}, z_{2} \in \mathbb{D}$ if and only if $f(z)$ is a Möbius transformation.

In particular,

$$
\left|f^{\prime}(z)\right| \leq \frac{1-|f(z)|^{2}}{1-|z|^{2}}, \quad \text { for } z \in \mathbb{D}
$$

and the equality holds for some $z \in \mathbb{D}$, if and only if $f(z)$ is a Möbius transformation.
Lemma 2 (see [29]). Suppose $f(z)=\sum_{k=0}^{\infty} a_{k} z^{k} \in \mathbb{H}$, then $\left|a_{k}\right| \leq 1-\left|a_{0}\right|^{2}$, for $k \in \mathbb{N}$.

Lemma 3. For $0 \leq x \leq x_{0} \leq 1$, it holds that $f(x):=x+A\left(1-x^{2}\right) \leq f\left(x_{0}\right), \quad$ whenever $0 \leq A \leq \frac{1}{2}$.

The proof is simple. We omit it.

Lemma 4 (see [28]). Suppose $f(z)=\sum_{k=0}^{\infty} a_{k} z^{k} \in \mathbb{H}$, and $a:=\left|a_{0}\right|$, then

$$
\begin{aligned}
& \sum_{k=0}^{\infty}\left|a_{k} \| z\right|^{k}+\left(\frac{1}{1+a}+\frac{|z|}{1-|z|}\right) \sum_{k=1}^{\infty}\left|a_{k}\right|^{2}|z|^{2 k} \\
& \leq a+\left(1-a^{2}\right) \frac{|z|}{1-|z|}, \quad \text { for }|z| \in[0,1) .
\end{aligned}
$$

Let $\mathbb{S}_{m}:=\left\{\omega \in \mathbb{H}: \omega(0)=\cdots=\omega^{(m-1)}(0)=0\right.$, and $\omega^{(m)}$ $(0) \neq 0\}, m \in \mathbb{N}=\{1,2,3, \ldots\}$, be the classes of Schwarz function $[30,31]$. The aim of this paper is to establish some new versions of Bohr-type inequalities with one parameter or involving convex combination for bounded analytic functions of Schwarz function. Theorems 1-4 are generalized. All of our results are sharp.

\section{Main Results}

Theorem 5. Suppose $f(z)=\sum_{k=0}^{\infty} a_{k} z^{k} \in \mathbb{H}, a:=\left|a_{0}\right|$ and $\omega_{m} \in \mathbb{S}_{m}, \psi_{n} \in \mathbb{S}_{n}$ for $m, n \in \mathbb{N}$. Then, for $\lambda \in(0,+\infty)$ and $N, s \in \mathbb{N}$, we have

$$
\left|f\left(\omega_{m}(z)\right)\right|+\lambda \sum_{k=N}^{\infty}\left|a_{s k} \| \psi_{n}(z)\right|^{s k} \leq 1,
$$

for $|z|=r \leq R_{\lambda, N, m, n, s}$, where $R_{\lambda, N, m, n, s}$ is the unique root in $(0,1)$ of the equation

$$
2 \lambda r^{N n s+m}+2 \lambda r^{N n s}+r^{m}+r^{n s}-r^{m+n s}-1=0,
$$

and the radius $R_{\lambda, N, m, n, s}$ is the best possible.

Proof. By the Schwarz lemma and the Schwarz-Pick lemma, respectively, we obtain

$$
\begin{gathered}
\left|\omega_{m}(z)\right| \leq|z|^{m}, \\
\left|\psi_{n}(z)\right| \leq|z|^{n}, \\
\left|f\left(\omega_{m}(z)\right)\right| \leq \frac{a+r^{m}}{1+a r^{m}}, \quad \text { for } z \in D .
\end{gathered}
$$

Then, by Lemma 2, we obtain

$$
\begin{gathered}
\left|f\left(\omega_{m}(z)\right)\right|+\lambda \sum_{k=N}^{\infty}\left|a_{s k} \| \psi_{n}(z)\right|^{s k} \leq \frac{a+r^{m}}{1+a r^{m}}+\lambda\left(1-a^{2}\right) \frac{r^{N n s}}{1-r^{n s}} \\
:=A_{N, m, n, s}(a, r, \lambda) .
\end{gathered}
$$


We just need to show that $A_{N, m, n, s}(a, r, \lambda) \leq 1$ holds for $r \leq R_{\lambda, N, m, n, s}$. That is to prove $A(a, r, \lambda) \leq 0$ for $r \leq R_{\lambda, N, m, n, s}$, where

$$
\begin{aligned}
A(a, r, \lambda) & =\left(a+r^{m}\right)\left(1-r^{n s}\right)+\lambda\left(1-a^{2}\right) r^{N n s}\left(1+a r^{m}\right)-\left(1+a r^{m}\right)\left(1-r^{n s}\right) \\
& =\left(a+r^{m}-1-a r^{m}\right)\left(1-r^{n s}\right)+\lambda\left(1-a^{2}\right) r^{N n s}\left(1+a r^{m}\right) \\
& =(1-a)\left[\left(r^{m}-1\right)\left(1-r^{n s}\right)+\lambda(1+a) r^{N n s}\left(1+a r^{m}\right)\right] \\
& \leq(1-a)\left[\left(r^{m}-1\right)\left(1-r^{n s}\right)+2 \lambda r^{N n s}\left(1+r^{m}\right)\right] .
\end{aligned}
$$

Obviously, it is enough to show that $\left(r^{m}-1\right)\left(1-r^{n s}\right)+$ $2 \lambda r^{N n s}\left(1+r^{m}\right) \leq 0$ holds for $r \leq R_{\lambda, N, m, n, s}$. Let

$$
\begin{aligned}
g(r) & =\left(r^{m}-1\right)\left(1-r^{n s}\right)+2 \lambda r^{N n s}\left(1+r^{m}\right) \\
& =2 \lambda r^{N n s+m}+2 \lambda r^{N n s}+r^{m}+r^{n s}-r^{m+n s}-1 .
\end{aligned}
$$

Then, we have

$$
\begin{aligned}
g^{\prime}(r)= & 2 \lambda(N n s+m) r^{N n s+m-1}+2 \lambda N n s r^{N n s-1} \\
& +m r^{m-1}+n s r^{n s-1}-(m+n s) r^{m+n s-1} \\
= & 2 \lambda(N n s+m) r^{N n s+m-1}+2 \lambda N n s r^{N n s-1} \\
& +m r^{m-1}\left(1-r^{n s}\right)+n s r^{n s-1}\left(1-r^{m}\right)>0 .
\end{aligned}
$$

We claim that $g(r)$ is an increasing function of $r \in(0,1)$ for fixing $\lambda \in(0,+\infty), N \in \mathbb{N}$. Meanwhile, we observe that $g(0)=-1<0$ and $g(1)=4 \lambda>0$. Then, there is a unique root $r^{*} \in(0,1)$ such that $g\left(r^{*}\right)=0$. Hence, $g(r) \leq 0$ holds for $r \leq r^{*}:=R_{\lambda, N, m, n, s}$.
Now, we show that the radius $R_{\lambda, N, m, n, s}$ is the best possible. Let $a \in[0,1)$,

$$
\begin{aligned}
\omega_{m}(z) & =z^{m}, \psi_{n}(z)=z^{n}, f(z)=\frac{a+z}{1+a z} \\
& =a+\left(1-a^{2}\right) \sum_{k=1}^{\infty}(-a)^{k-1} z^{k}, z \in D .
\end{aligned}
$$

Taking $z=r \in(0,1)$, then the left side of inequality (15) reduces to

$$
\left|f\left(r^{m}\right)\right|+\lambda \sum_{k=N}^{\infty}\left|a_{s k}\right| r^{n s k}=\frac{a+r^{m}}{1+a r^{m}}+\lambda\left(1-a^{2}\right) \frac{a^{N s-1} r^{N n s}}{1-a^{s} r^{n s}}
$$

Now, we just need to show that if $r>R_{\lambda, N, m, n, s}$, then there exists an $a \in[0,1)$ such that the right side of $(23)$ is greater than 1 . That is to prove $l(a, r, \lambda)>0$ for $r>R_{\lambda, N, m, n, s}$, where

$$
\begin{aligned}
l(a, r, \lambda) & =\left(a+r^{m}\right)\left(1-a^{s} r^{n s}\right)+\lambda\left(1-a^{2}\right) a^{N s-1} r^{N n s}\left(1+a r^{m}\right)-\left(1+a r^{m}\right)\left(1-a^{s} r^{n s}\right) \\
& =\left(1-a^{s} r^{n s}\right)\left(a+r^{m}-1-a r^{m}\right)+\lambda\left(1-a^{2}\right) a^{N s-1} r^{N n s}\left(1+a r^{m}\right) \\
& =(1-a)\left[\left(r^{m}-1\right)\left(1-a^{s} r^{n s}\right)+\lambda(1+a) a^{N s-1} r^{N n s}\left(1+a r^{m}\right)\right] .
\end{aligned}
$$

Let

$$
\begin{aligned}
j(a, r, \lambda)= & \left(r^{m}-1\right)\left(1-a^{s} r^{n s}\right) \\
& +\lambda(1+a) a^{N s-1} r^{N n s}\left(1+a r^{m}\right) .
\end{aligned}
$$

Observe that

$$
\begin{aligned}
\lim _{a \rightarrow 1^{-}} j(a, r, \lambda)= & 2 \lambda r^{N n s+m}+2 \lambda r^{N n s} \\
& +r^{n s}+r^{m}+-r^{n s+m}-1=g(r) .
\end{aligned}
$$

Thus, the monotonicity of $g(r)$ implies that if $r>R_{\lambda, N, m, n, s}$, then $g(r)>0$. Therefore, by the continuity of $j(a, r, \lambda)$ for variable $a$, we have that if $r>R_{\lambda, N, m, n, s}$, there exists an $a \in[0,1)$ such that $j(a, r, \lambda)>0$, so does $l(a, r, \lambda)$.
In Theorem 5, setting $s=1$, then we have Corollary 1 .

Corollary 1. Suppose $f(z)=\sum_{k=0}^{\infty} a_{k} z^{k} \in \mathbb{H}, a:=\left|a_{0}\right|$ and $\omega_{m} \in \mathbb{S}_{m}, \psi_{n} \in \mathbb{S}_{n}$ for $m, n \in \mathbb{N}$. Then, for $\lambda \in(0,+\infty)$ and $N \in \mathbb{N}$, it holds that

$$
\left|f\left(\omega_{m}(z)\right)\right|+\lambda \sum_{k=N}^{\infty}\left|a_{k} \| \psi_{n}(z)\right|^{k} \leq 1,
$$

for $|z|=r \leq R_{\lambda, N, m, n}$, where $R_{\lambda, N, m, n}$ is the unique root in $(0,1)$ of the equation

$$
2 \lambda r^{N n+m}+2 \lambda r^{N n}+r^{m}+r^{n}-r^{m+n}-1=0,
$$

and the radius $R_{\lambda, N, m, n}$ is the best possible. 


$$
\lambda r^{N n s+m}+\lambda r^{N n s}+r^{n s}+r^{m}-r^{n s+m}-1=0
$$

(1) If $\omega_{m}(z)=\psi_{n}(z)=z, N=1$, and $\lambda=1$ in Corollary 1 , then it reduces to the first part of Theorem 1 .

(2) If $\omega_{m}(z)=\psi_{n}(z)=z, N=1$, and $\lambda=1$ in Theorem 5, then it reduces to Theorem 2 .

Theorem 6. Suppose $f(z)=\sum_{k=0}^{\infty} a_{k} z^{k} \in \mathbb{H}, a:=\left|a_{0}\right|$ and $\omega_{m} \in \mathbb{S}_{m}, \psi_{n} \in \mathbb{S}_{n}$ for $m, n \in \mathbb{N}$. Then, for $\lambda \in(0,+\infty)$ and $N \in \mathbb{N}$, we have

$$
\left|f\left(\omega_{m}(z)\right)\right|^{2}+\lambda \sum_{k=N}^{\infty}\left|a_{s k} \| \psi_{n}(z)\right|^{s k} \leq 1,
$$

for $|z|=r \leq R_{\lambda, N, m, n, s}^{*}$, where $R_{\lambda, N, m, n, s}^{*}$ is the unique root in $(0,1)$ of the equation and the radius $R_{\lambda, N, m, n, s}^{*}$ is the best possible.

Proof. Inequality (17) and Lemma 2 lead to that

$$
\begin{aligned}
& \left|f\left(\omega_{m}(z)\right)\right|^{2}+\lambda \sum_{k=N}^{\infty}\left|a_{s k} \| \psi_{n}(z)\right|^{s k} \\
& \leq\left(\frac{a+r^{m}}{1+a r^{m}}\right)^{2}+\lambda\left(1-a^{2}\right) \frac{r^{N n s}}{1-r^{n s}}:=B_{N, m, n, s}(a, r, \lambda) .
\end{aligned}
$$

We just need to show that $B_{N, m, n, s}(a, r, \lambda) \leq 1$ holds for $r \leq R_{\lambda, N, m, n, s}^{*}$. That is to prove $B(a, r, \lambda) \leq 0$ for $r \leq R_{\lambda, N, m, n, s}^{*}$, where

$$
\begin{aligned}
B(a, r, \lambda) & =\left(a+r^{m}\right)^{2}\left(1-r^{n s}\right)+\lambda\left(1-a^{2}\right) r^{N n s}\left(1+r^{m}\right)^{2}-\left(1+a r^{m}\right)^{2}\left(1-r^{n s}\right) \\
& =\left(1-r^{n s}\right)\left(a+r^{m}+1+a r^{m}\right)\left(a+r^{m}-1-a r^{m}\right)+\lambda\left(1-a^{2}\right) r^{N n s}\left(1+a r^{m}\right)^{2} \\
& =\left(1-a^{2}\right)\left[\left(1-r^{n s}\right)\left(1+r^{m}\right)\left(r^{m}-1\right)+\lambda r^{N n s}\left(1+a r^{m}\right)\right]^{2} \\
& \leq\left(1-a^{2}\right)\left(1+r^{m}\right)\left[\left(1-r^{n s}\right)\left(r^{m}-1\right)+\lambda r^{N n s}\left(1+r^{m}\right)\right] .
\end{aligned}
$$

It is sufficient for us to prove $\left(1-r^{n s}\right)\left(r^{m}-1\right)+\lambda r^{N n s}\left(1+r^{m}\right) \leq 0$ holds for $r \leq R_{\lambda, N, m, n, s}^{*}$. Let

$$
\begin{aligned}
g(r) & =\left(1-r^{n s}\right)\left(r^{m}-1\right)+\lambda r^{N n s}\left(1+r^{m}\right) \\
& =\lambda r^{N n s+m}+\lambda r^{N n s}+r^{m}+r^{n s}-r^{n s+m}-1,
\end{aligned}
$$

then we have

$$
\begin{aligned}
g^{\prime}(r)= & \lambda(N n s+m) r^{N n s+m-1}+\lambda N n s r^{N n s-1} \\
& +m r^{m-1}+n s r^{n s-1}-(m+n s) r^{m+n s-1} \\
= & \lambda(N n s+m) r^{N n s+m-1}+\lambda N n s r^{N n s-1} \\
& +n s r^{n s-1}\left(1-r^{m}\right)+m r^{m-1}\left(1-r^{n s}\right)>0 .
\end{aligned}
$$

$$
\begin{aligned}
l(a, r, \lambda) & =\left(a+r^{m}\right)^{2}\left(1-a r^{n s}\right)+\lambda\left(1-a^{2}\right) a^{N s-1} r^{N n s}\left(1+a r^{m}\right)^{2}-\left(1+a r^{m}\right)^{2}\left(1-a r^{n s}\right) \\
& =\left(1-a r^{n s}\right)\left(a+r^{m}+1+a r^{m}\right)\left(a+r^{m}-1-a r^{m}\right)+\lambda\left(1-a^{2}\right) a^{N s-1} r^{N n s}\left(1+a r^{m}\right)^{2} \\
& =\left(1-a^{2}\right)\left(1+r^{m}\right)\left[\left(r^{m}-1\right)\left(1-a r^{n s}\right)+\lambda a^{N s-1} r^{N n s}\left(1+a r^{m}\right)\right] .
\end{aligned}
$$

Let

$$
\begin{aligned}
j(a, r, \lambda) & =\left(r^{m}-1\right)\left(1-a r^{n s}\right)+\lambda a^{N s-1} r^{N n s}\left(1+a r^{m}\right) \\
& =\lambda r^{N n+m} a^{N s}+\lambda r^{N n s} a^{N s-1}+r^{m}+a r^{n s}-a r^{n s+m}-1 .
\end{aligned}
$$$$
\text { (37) }
$$

Obviously, $g(r)$ is an increasing function of $r \in(0,1)$ for fix $\lambda \in(0,+\infty), N, s \in \mathbb{N}$, and we also have $g(0)=-1<0$, $g(1)=2 \lambda>0$. Then, there is a unique root $r^{*} \in(0,1)$ such that $g\left(r^{*}\right)=0$. Hence, $g(r) \leq 0$ holds for $r \leq r^{*}:=R_{\lambda, N, m, n, s^{*}}^{*}$

Now, we show that the radius $R_{\lambda, N, m, n, s}^{*}$ is the best possible. We still consider the functions $\omega_{m}(z), \psi_{n}(z), f(z)$ as in (22). Taking $z=r \in(0,1)$, then the left side of inequality (29) reduces to

$$
\left|f\left(r^{m}\right)\right|^{2}+\lambda \sum_{k=N}^{\infty}\left|a_{s k}\right| r^{n s k}=\left(\frac{a+r^{m}}{1+a r^{m}}\right)^{2}+\lambda\left(1-a^{2}\right) \frac{a^{N s-1} r^{N n s}}{1-a r^{n s}} .
$$

Now, we just need to show that if $r>R_{\lambda, N, m, n, s}^{*}$, then there exists an $a \in[0,1)$ such that the right side of (35) is greater than 1 . That is to prove $l(a, r, \lambda)>0$, where
Observe that

$$
\begin{aligned}
\lim _{a \rightarrow 1^{-}} j(a, r, \lambda)= & \lambda r^{N n+m}+\lambda r^{N n s}+r^{m} \\
& +r^{n s}-r^{n s+m}-1=g(r) .
\end{aligned}
$$


Thus, the monotonicity of $g(r)$ implies that if $r>R_{\lambda, N, m, n, s}^{*}$, then $g(r)>0$. Therefore, by the continuity of $j(a, r, \lambda)$ for variable $a$, we have that if $r>R_{\lambda, N, m, n, s}^{*}$ there exists an $a \in[0,1)$ such that inequality $j(a, r, \lambda)>0$, so does $l(a, r, \lambda)$. 2

In Theorem 6 , setting $s=1$, then we have Corollary

Corollary 2. Suppose $f(z)=\sum_{k=0}^{\infty} a_{k} z^{k} \in \mathbb{U}, a:=\left|a_{0}\right|$ and $\omega_{m} \in \mathbb{S}_{m}, \psi_{n} \in \mathbb{S}_{n}$ for $m, n \in \mathbb{N}$. Then, for $\lambda \in(0,+\infty)$ and $N \in \mathbb{N}$, we have

$$
\left|f\left(\omega_{m}(z)\right)\right|^{2}+\lambda \sum_{k=N}^{\infty}\left|a_{k} \| \psi_{n}(z)\right|^{k} \leq 1,
$$

for $|z|=r \leq R_{\lambda, N, m, n}^{*}$, where $R_{\lambda, N, m, n}$ is the unique root in $(0,1)$ of the equation

$$
\lambda r^{N n+m}+\lambda r^{N n}+r^{n s}+r^{m}-r^{n+m}-1=0,
$$

and the radius $R_{\lambda, N, m, n}^{*}$ is the best possible.

Remark 2. If $\omega_{m}(z)=\psi_{n}(z)=z$ and $\lambda=1$ in Corollary 2, then it reduces to the second part of Theorem 1 .

Theorem 7. Suppose $f(z)=\sum_{k=0}^{\infty} a_{k} z^{k} \in \mathbb{H}, a:=\left|a_{0}\right|$, and $\omega_{m} \in \mathbb{S}_{m}$ for $m \in \mathbb{N}$. Then, for $\lambda \in(0,+\infty)$ and $N \in \mathbb{N}$, we have

$$
\left|f\left(\omega_{m}(z)\right)\right|+\left|f^{\prime}\left(\omega_{m}(z)\right)\left\|\omega_{m}(z)\left|+\lambda \sum_{k=N}^{\infty}\right| a_{k}\right\| \omega_{m}(z)\right|^{k} \leq 1,
$$

for $|z|=r \leq R_{\lambda, N, m}$, where $R_{\lambda, N, m}$ is the unique root in $(0, \sqrt[m]{\sqrt{2}-1})$ of the equation

$$
2 \lambda r^{N m}\left(1+r^{m}\right)^{2}+\left(1-r^{m}\right)\left(r^{2 m}+2 r^{m}-1\right)=0,
$$

and the radius $R_{\lambda, N, m}$ is the best possible.

Proof. By the Schwarz lemma and the Schwarz-Pick lemma, respectively, we have

$$
\begin{gathered}
\left|\omega_{m}(z)\right| \leq r^{m}, \\
\left|f\left(\omega_{m}(z)\right)\right| \leq \frac{r^{m}+a}{1+a r^{m}}, \quad \text { for } z \in \mathbb{D} .
\end{gathered}
$$

Then, by Lemmas $1-3$, we obtain

$$
\begin{aligned}
& \left|f\left(\omega_{m}(z)\right)\right|+\left|f^{\prime}\left(\omega_{m}(z)\right)\right|\left|\omega_{m}(z)\right|+\lambda \sum_{k=N}^{\infty}\left|a_{k} \| \omega_{m}(z)\right|^{k} \\
& \leq\left|f\left(\omega_{m}(z)\right)\right|+\frac{1-\left|f\left(\omega_{m}(z)\right)\right|^{2}}{1-\left|\omega_{m}(z)\right|^{2}}\left|\omega_{m}(z)\right|+\lambda \sum_{k=N}^{\infty}\left|a_{k} \| \omega_{m}(z)\right|^{k} \\
& \leq \frac{a+r^{m}}{1+a r^{m}}+\left[1-\left(\frac{a+r^{m}}{1+a r^{m}}\right)^{2}\right] \frac{r^{m}}{1-r^{2 m}}+\lambda\left(1-a^{2}\right) \frac{r^{N m}}{1-r^{m}} \\
& =\frac{a+r^{m}}{1+a r^{m}}+\frac{\left(1-a^{2}\right) r^{m}}{\left(1+a r^{m}\right)^{2}}+\lambda\left(1-a^{2}\right) \frac{r^{N m}}{1-r^{m}}:=C_{N, m}(a, r, \lambda),
\end{aligned}
$$

for $r \leq \sqrt[m]{\sqrt{2}-1}$, where $\sqrt[m]{\sqrt{2}-1}$ is the unique root of the equation $r^{2 m}+2 r^{m}-1=0$ in $(0,1)$. We just need to show that $A_{N, m}(a, r, \lambda) \leq 1$ holds for $r \leq R_{\lambda, N, m}$. That is to prove $C(a, r, \lambda) \leq 0$ for $r \leq R_{\lambda, N, m}$, where

$$
\begin{aligned}
C(a, r, \lambda) & =\left[\left(a+r^{m}\right)\left(1+a r^{m}\right)+\left(1-a^{2}\right) r^{m}-\left(1+a r^{m}\right)^{2}\right]\left(1-r^{m}\right)+\lambda\left(1-a^{2}\right) r^{N m}\left(1+a r^{m}\right)^{2} \\
& =\left(1-r^{m}\right)\left(1+a r^{m}\right)\left(a+r^{m}-1-a r^{m}\right)+\left(1-a^{2}\right) r^{m}\left(1-r^{m}\right)+\lambda\left(1-a^{2}\right) r^{N m}\left(1+a r^{m}\right)^{2} \\
& =(1-a)\left[\left(1-r^{m}\right)\left(1+a r^{m}\right)\left(r^{m}-1\right)+(1+a) r^{m}\left(1-r^{m}\right)+\lambda(1+a) r^{N m}\left(1+a r^{m}\right)^{2}\right] .
\end{aligned}
$$

Let

$$
\begin{aligned}
g(r)= & \left(1-r^{m}\right)\left(1+a r^{m}\right)\left(r^{m}-1\right)+(1+a) r^{m}\left(1-r^{m}\right) \\
& +\lambda(1+a) r^{N m}\left(1+a r^{m}\right)^{2},
\end{aligned}
$$

$$
\begin{aligned}
g(r) & =\lambda r^{N m+2 m} a^{3}+\lambda r^{N m}\left(r^{2 m}+2 r^{m}\right) a^{2}+\left[\left(1-r^{m}\right) r^{2 m}+\lambda r^{N m}\left(2 r^{m}+1\right)\right] a+\left(1-r^{m}\right)\left(2 r^{m}-1\right)+\lambda r^{N m} \\
& \leq \lambda r^{N m+2 m}+\lambda r^{N m}\left(r^{2 m}+2 r^{m}\right)+\left(1-r^{m}\right) r^{2 m}+\lambda r^{N m}\left(2 r^{m}+1\right)+\left(1-r^{m}\right)\left(2 r^{m}-1\right)+\lambda r^{N m} \\
& =2 \lambda r^{N m}\left(r^{m}+1\right)^{2}+\left(1-r^{m}\right)\left(r^{2 m}+2 r^{m}-1\right):=h(r) .
\end{aligned}
$$


It is easy to verify that both $2 \lambda r^{N m}\left(r^{m}+1\right)^{2}$ and $(1-$ $\left.r^{m}\right)\left(r^{2 m}+2 r^{m}-1\right)$ are continuous and increasing functions for $r \in[0, \sqrt[m]{\sqrt{2}-1}]$. Thus, $h(r)$ is a monotonically increasing function for $r \in[0, \sqrt[m]{\sqrt{2}-1}]$. Furthermore, noting $h(0)=-1<0$ and $h(\sqrt[m]{\sqrt{2}-1})=4 \lambda(\sqrt{2}-1)^{N}>0$, thus there exists a unique real root $r^{*} \in(0, \sqrt[m]{\sqrt{2}-1})$ such that $h\left(r^{*}\right)=0$. Hence, it holds that $h(r) \leq 0$, for $r \leq r^{*}:=R_{\lambda, N, m}$.

Now, we show that the radius $R_{\lambda, N, m}$ is the best possible. Let $a \in[0,1)$,

$$
\begin{aligned}
\omega_{m}(z) & =z^{m}, \\
f(z) & =\frac{a+z}{1+a z}=a+\left(1-a^{2}\right) \sum_{k=1}^{\infty}(-a)^{k-1} z^{k}, \quad z \in \mathbb{D} .
\end{aligned}
$$

Taking $z=r \in(0,1)$, then the left side of inequality (41) reduces to

$$
\begin{aligned}
\left|f\left(r^{m}\right)\right|+\left|f^{\prime}\left(r^{m}\right) \| r^{m}\right|+\lambda \sum_{k=N}^{\infty}\left|a_{k}\right| r^{m k}= & \frac{a+r^{m}}{1+a r^{m}}+\frac{\left(1-a^{2}\right) r^{m}}{\left(1+a r^{m}\right)^{2}} \\
& +\lambda\left(1-a^{2}\right) \frac{a^{N-1} r^{N m}}{1-a r^{m}} .
\end{aligned}
$$

Now, we just need to show that if $r>R_{\lambda, N, m}$, then there exists an $a \in[0,1)$ such that the right side of $(49)$ is greater than 1 . That is to prove $l(a, r, \lambda)>0$ for $r>R_{\lambda, N, m}$, where

$$
\begin{aligned}
l(a, r, \lambda)= & \left(1-a r^{m}\right)\left(1+a r^{m}\right)\left(a+r^{m}-1-a r^{m}\right)+\left(1-a^{2}\right) \\
& {\left[r^{m}\left(1-a r^{m}\right)+\lambda a^{N-1} r^{N m}\left(1+a r^{m}\right)^{2}\right]=(1-a) } \\
& {\left[\left(r^{m}-1\right)\left(1-a r^{m}\right)\left(1+a r^{m}\right)+(1+a) r^{m}\left(1-a r^{m}\right)+\lambda(1+a) a^{N-1} r^{N m}\left(1+a r^{m}\right)^{2}\right] . }
\end{aligned}
$$

Let

$$
\begin{aligned}
j(a, r, \lambda)= & \left(r^{m}-1\right)\left(1-a r^{m}\right)\left(1+a r^{m}\right)+(1+a) r^{m}\left(1-a r^{m}\right) \\
& +\lambda(1+a) a^{N-1} r^{N m}\left(1+a r^{m}\right)^{2} .
\end{aligned}
$$

Observe that

$$
\begin{aligned}
\lim _{a \longrightarrow 1^{-}} j(a, r, \lambda)= & 2 \lambda r^{N m}\left(1+r^{m}\right)^{2} \\
& +\left(1-r^{m}\right)\left(r^{2 m}+2 r^{m}-1\right)=h(r) .
\end{aligned}
$$

Thus, the monotonicity of $h(r)$ implies that if $r \in\left(R_{\lambda, N, m}, \sqrt[m]{\sqrt{2}-1}\right)$, then $h(r)>0$. Therefore, by the continuity of $j(a, r, \lambda)$ for variable $a$, we have that if $r \in\left(R_{\lambda, N, m}, \sqrt[m]{\sqrt{2}-1}\right)$, there exists an $a \in[0,1)$ such that inequality $j(a, r, \lambda)>0$, so does $l(a, r, \lambda)$.

Remark 3. If $\omega_{m}(z)=z, N=2$, and $\lambda=1$ in Theorem 7, then it reduces to Theorem 3 .

Theorem 8. Suppose $f(z)=\sum_{k=0}^{\infty} a_{k} z^{k} \in \mathbb{U}, a:=\left|a_{0}\right|$, and $\omega_{m} \in \mathbb{S}_{m}$, for $m \in \mathbb{N}$. Then, for $t \in[0,1)$, we have

$$
t\left|f\left(\omega_{m}(z)\right)\right|+(1-t)\left[\sum_{k=0}^{\infty}\left|a_{k} \| \omega_{m}(z)\right|^{k}+\left(\frac{1}{1+a}+\frac{\left|\omega_{m}(z)\right|}{1-\left|\omega_{m}(z)\right|}\right) \sum_{k=1}^{\infty}\left|a_{k}\right|^{2}\left|\omega_{m}(z)\right|^{2 k}\right] \leq 1,
$$

for $|z|=r \leq R_{t, m}$, where

$$
R_{t, m}= \begin{cases}\sqrt[m]{\frac{1}{(1+a) \sqrt{1-t}+1},}, & \text { for } t \in\left[0, \frac{(1+a)^{2}-1}{(1+a)^{2}}\right) \cup\left(\frac{(1+a)^{2}-1}{(1+a)^{2}}, 1\right), \\ \sqrt[m]{\frac{1}{2},} & \text { for } t=\frac{(1+a)^{2}-1}{(1+a)^{2}},\end{cases}
$$




$$
\begin{aligned}
& t\left|f\left(\omega_{m}(z)\right)\right|+(1-t)\left[\sum_{k=0}^{\infty}\left|a_{k} \| \omega_{m}(z)\right|^{k}+\left(\frac{1}{1+a}+\frac{\left|\omega_{m}(z)\right|}{1-\left|\omega_{m}(z)\right|}\right) \sum_{k=1}^{\infty}\left|a_{k}\right|^{2}\left|\omega_{m}(z)\right|^{2 k}\right] \\
& \leq t \frac{a+r^{m}}{1+a r^{m}}+(1-t) a+(1-t)\left(1-a^{2}\right) \frac{r^{m}}{1-r^{m}}:=D_{m}(a, r, t) .
\end{aligned}
$$

We just need to show that $D_{m}(a, r, t) \leq 1$ holds for $r \leq R_{t, m}$. That is to prove $D(a, r, t) \leq 0$ for $r \leq R_{t, m}$, where

$$
\begin{aligned}
D(a, r, t)= & t\left(a+r^{m}\right)\left(1-r^{m}\right)+(1-t)\left(1+a r^{m}\right) \\
& {\left[a\left(1-r^{m}\right)+\left(1-a^{2}\right) r^{m}\right]-\left(1+a r^{m}\right)\left(1-r^{m}\right) } \\
= & (1-a)\left\{\left[-(1+a)^{2} t+(1+a)^{2}-1\right] r^{2 m}+2 r^{m}-1\right\} .
\end{aligned}
$$

Let

$$
g(r)=\left[-(1+a)^{2} t+(1+a)^{2}-1\right] r^{2 m}+2 r^{m}-1 .
$$

Then, we divide it into two cases to discuss.
Case 1. If $t \in\left[0,\left((1+a)^{2}-1 /(1+a)^{2}\right)\right) \cup\left(\left((1+a)^{2}\right.\right.$ $\left.\left.-1 /(1+a)^{2}\right), 1\right)$, then we have $g(r) \leq 0$ for $r \leq$ $\sqrt[m]{1 /((1+a) \sqrt{1-t}+1)}$, where $\sqrt[m]{1 /((1+a) \sqrt{1-t}+1)}$ is the unique root in $(0,1)$ of equation $\left[-(1+a)^{2} t+(1+a)^{2}-1\right] r^{2 m}+2 r^{m}-1=0 . \quad$ Thus, $B(a, r, t) \leq 0$ for $r \leq \sqrt[m]{1 /((1+a) \sqrt{1-t}+1)}$.

Case 2. If $t=(1+a)^{2}-1 /(1+a)^{2}$, then we have $g(r) \leq 0$ for $r \leq \sqrt[m]{1 / 2}$. Thus, $B(a, r, t) \leq 0$ for $r \leq \sqrt[m]{1 / 2}$.

Now, we show that the radius $R_{t, m}$ is the best possible. Let $a \in[0,1)$; we still consider the functions $\omega_{m}(z), f(z)$ as in (48). Taking $z=r \in(0,1)$, then the left side of inequality (53) reduces to

$$
\begin{aligned}
& t\left|f\left(r^{m}\right)\right|+(1-t)\left[\sum_{k=0}^{\infty}\left|a_{k}\right| r^{m k}+\left(\frac{1}{1+a}+\frac{r^{m}}{1-r^{m}}\right) \sum_{k=1}^{\infty}\left|a_{k}\right|^{2} r^{2 m k}\right] \\
& =t \frac{a+r^{m}}{1+a r^{m}}+(1-t)\left[a+\sum_{k=1}^{\infty}\left(1-a^{2}\right) a^{k-1} r^{m k}+\left(\frac{1}{1+a}+\frac{r^{m}}{1-r^{m}}\right) \sum_{k=1}^{\infty}\left(1-a^{2}\right)^{2} a^{2 k-2} r^{2 m k}\right] \\
& =t \frac{a+r^{m}}{1+a r^{m}}+(1-t)\left[a+\left(1-a^{2}\right) \frac{r^{m}}{1-r^{m}}\right]:=D_{m}^{*}(a, r, t) .
\end{aligned}
$$

Now, we just need to show that if $r>R_{t, m}$, then there exists an $a \in[0,1)$ such that $D_{m}^{*}(a, r, t)$ is greater than 1 . That is to prove $l(a, r, t)>0$ for $r>R_{t, m}$, where

$$
\begin{aligned}
l(a, r, t)= & t\left(a+r^{m}\right)\left(1-r^{m}\right)+(1-t) \\
& {\left[a\left(1+a r^{m}\right)\left(1-r^{m}\right)+r^{m}\left(1+a r^{m}\right)\right] } \\
& -\left(1+a r^{m}\right)\left(1-r^{m}\right) \\
= & (1-a)\left\{\left[-(1+a)^{2} t+(1+a)^{2}-1\right] r^{2 m}+2 r^{m}-1\right\} .
\end{aligned}
$$

Let

$$
h(r)=\left[-(1+a)^{2} t+(1+a)^{2}-1\right] r^{2 m}+2 r^{m}-1 .
$$

Next, we divide it into two cases to discuss.

Case 1. If $t \in\left[0,\left(\left((1+a)^{2}-1\right) /(1+a)^{2}\right)\right) \cup\left((1+a)^{2}\right.$ $\left.-1 /(1+a)^{2}, 1\right)$, then we have $h(r) \geq 0$ for $r \geq \sqrt[m]{1 /((1+a) \sqrt{1-t}+1)}$. Thus, $\quad l(a, r, t) \geq 0$ for $r \geq \sqrt[m]{1 /((1+a) \sqrt{1-t}+1)}$
Case 2. If $t=\left((1+a)^{2}-1\right) /(1+a)^{2}$, then we have $h(r)$ $\geq 0$ for $r \geq \sqrt[m]{1 / 2}$. Thus, $l(a, r, t) \geq 0$ for $r \geq \sqrt[m]{1 / 2}$.

Remark 4. If $\omega_{m}(z)=z$ and $t=0$ in Theorem 8 , then it reduces to Theorem 4 .

\section{Conclusion}

We establish some new versions of Bohr-type inequalities with one parameter or involving convex combination for bounded analytic functions of Schwarz function, and we conclude that all of the corresponding Bohr radii in the paper are exact. These inequalities generalize some earlier results on the Bohr radius problem.

\section{Data Availability}

The author declares that this research is purely theoretical and does not associate with any data. 


\section{Conflicts of Interest}

The author declares that there are no conflicts of interest.

\section{Acknowledgments}

The work was supported by Anhui Province Training Programs for the First-class Talents (2019rcsfjd088) and Teaching-Group Program of Anhui Province (2020kcszjxtd57).

\section{References}

[1] H. Bohr, "A theorem concerning power series," Proceedings of the London Mathematical Society, vol. 13, no. 2, pp. 1-5, 1914.

[2] V. I. Paulsen, G. Popascu, and D. Singh, "On Bohr's inequality," Proceedings of the London Mathematical Society, vol. 3, no. 85, pp. 493-512, 2002.

[3] S. Sidon, "Uber einen Satz von Herrn Bohr," Mathematische Zeitschrift, vol. 26, no. 1, pp. 731-732, 1927.

[4] M. Tomic, "Sur un théorême de H. Bohr," Mathematica Scandinavica, vol. 11, pp. 103-106, 1962.

[5] I. R. Kayumov and S. Ponnusamy, "Bohr inequality for odd analytic functions," Computational Methods and Function Theory, vol. 17, no. 4, pp. 679-688, 2017.

[6] B. Bhowmik and N. Das, "Bohr phenomenon for subordinating families of certain univalent functions," Journal of Mathematical Analysis and Applications, vol. 462, no. 2, pp. 1087-1098, 2018.

[7] Y. Huang, M. S. Liu, and S. Ponnusamy, "Refined Bohr-type inequalities with area measure for bounded analytic functions," Analysis and Mathematical Physics, vol. 10, 2020.

[8] X. J. Hu, Q. H. Wang, and B. Y. Long, "Bohr-type inequalities for bounded analytic functions of Schwarz functions," AIMS Mathematics, vol. 6, no. 12, pp. 13608-13621, 2021.

[9] X. J. Hu, Q. H. Wang, and B. Y. Long, "Bohr-type inequalities with one parameter for bounded analytic functions of Schwarz function," Bulletin of the Malaysian Mathematical Sciences Society, 2021.

[10] Y. Abu-Muhanna, R. M. Ali, and Z. C. Ng, "Bohr radius for the punctured disk," Mathematische Nachrichten, vol. 290, 2017.

[11] Y. Abu Muhanna, R. M. Ali, Z. C. Ng, and S. F. M. Hasni, "Bohr radius for subordinating families of analytic functions and bounded harmonic mappings," Journal of Mathematical Analysis and Applications, vol. 420, no. 1, pp. 124-136, 2014.

[12] R. M. Ali, R. W. Barnard, and A. Y. Solynin, "A note on Bohr's phenomenon for power series," Journal of Mathematical Analysis and Applications, vol. 449, no. 1, pp. 154-167, 2017.

[13] L. Aizenberg, "Generalization of results about the Bohr radius for power series," Studia Mathematica, vol. 180, no. 2, pp. 161-168, 2007.

[14] Y. Abu Muhanna and R. M. Ali, "Bohr's phenomenon for analytic functions into the exterior of a compact convex body," Journal of Mathematical Analysis and Applications, vol. 379, no. 2, pp. 512-517, 2011.

[15] Z. H. Liu and S. Ponnusamy, "Bohr radius for subordination and K-quasi conformal harmonic mappings," Bulletin of the Malaysian Mathematical Sciences Society, vol. 42, no. 5, pp. 2151-2168, 2019.

[16] P. B. Djakov and M. S. Ramanujan, "A remark on Bohr's theorems and its generalizations," The Journal of Analytical Chemistry, vol. 8, pp. 65-77, 2000.
[17] L. Aizenberg, Generalization of Carathéodory's Inequality and the Bohr Radius for Multidimensional Power Series, Birkh User, Basel, 2005.

[18] F. Bayart, D. Pellegrino, and J. B. Seoane-Sepúlveda, "The Bohr radius of the $n$-dimensional polydisk is equivalent to $(\log n) / n$," Advances in Mathematics, vol. 264, pp. 726-746, 2014.

[19] Y. Abu-Muhanna and G. Gunatillake, "Bohr phenomenon in weighted Hardy-Hilbert spaces," Acta Scientiarum Mathematicarum, vol. 78, no. 3-4, pp. 517-528, 2012.

[20] O. Blasco, "The Bohr radius of a Banach space," in Vector Measures, Integration and Related TopicsBirkhäuser Basel, Basel, 2010.

[21] V. I. Paulsen and D. Singh, "Bohr's inequality for uniform algebras," Proceedings of the American Mathematical Society, vol. 132, no. 12, pp. 3577-3579, 2004.

[22] R. M. Ali, Y. Abu-Muhanna, and S. Ponnusamy, "On the Bohr inequality, in progress in approximation theory and applicable complex analysis," Springer Optimization and Its Applications, vol. 117, pp. 265-295, 2016.

[23] S. Alkhaleefah, I. Kayumov, and S. Ponnusamy, "On the Bohr inequality with a fixed zero coefficient," Proceedings of the American Mathematical Society, vol. 147, no. 12, pp. 52635274, 2019.

[24] I. R. Kayumov and S. Ponnusamy, "Bohr's inequalities for the analytic functions with lacunary series and harmonic functions," Journal of Mathematical Analysis and Applications, vol. 465, no. 2, pp. 857-871, 2018.

[25] I. R. Kayumov and S. Ponnusamy, "Improved version of Bohr's inequality," Comptes Rendus Mathematique, vol. 356, no. 3, pp. 272-277, 2018.

[26] I. R. Kayumov and S. Ponnusamy, "Bohr-Rogosinski radius for analytic functions," 2017, https://arxiv.org/pdf/1708. 05585.pdf.

[27] M. S. Liu, Y. M. Shang, and J. F. Xu, "Bohr-type inequalities of analytic functions," Journal of Inequalities and Applications, vol. 345 , no. 13, 2018.

[28] S. Ponnusamy, R. Vijayakumar, and K. J. Wirths, "New inequalities for the coefficients of unimodular bounded functions," Results in Mathematics, vol. 75, no. 3, p. 107, 2020.

[29] I. Graham and G. Kohr, Geometric Function Theory in One and Higher Dimensions, Marcel Dekker, New York, NY, USA, 2003.

[30] P. Agarwal, S. S. Dragomir, M. Jleli, and B. Samet, Advances in Mathematical Inequalities and Applications, Birkh User, Basel, 2018.

[31] A. A. Attiya, A. M. Lashin, E. E. Ali, and P. Agarwal, "Coefficient bounds for certain classes of analytic functions associated with faber polynomial," Symmetry, vol. 13, no. 2, p. 302, 2021. 